\title{
IMPACT OF LEVEL OF DETAILS IN THE 3D RECONSTRUCTION OF TREES FOR MICROCLIMATE MODELING
}

\author{
E. Bournez ${ }^{\text {a,*, T. Landes }}{ }^{\text {a }}$, M. Saudreau ${ }^{\text {b }}$, P. Kastendeuch ${ }^{\text {a }}$, G. Najjar ${ }^{\text {a }}$ \\ ${ }^{\text {a }}$ ICube Laboratory UMR 7357, University of Strasbourg, CNRS, INSA Strasbourg, France - (elena.bournez, tania.landes)@insa- \\ strasbourg.fr; (georges.najjar, kasten)@unistra.fr \\ b PIAF Laboratory, UMR547 University Blaise Pascal, INRA Clermont-Ferrand, France - marc.saudreau@ clermont.inra.fr
}

\section{Commission VIII, WG VIII/3}

KEY WORDS: Urban climate, Trees, Terrestrial laser scanner, Point cloud, 3D reconstruction, Leaves, Evapotranspiration, Level of details.

\begin{abstract}
:
In the 21 st century, urban areas undergo specific climatic conditions like urban heat islands which frequency and intensity increase over the years. Towards the understanding and the monitoring of these conditions, vegetation effects on urban climate are studied. It appears that a natural phenomenon, the evapotranspiration of trees, generates a cooling effect in urban environment. In this work, a $3 \mathrm{D}$ microclimate model is used to quantify the evapotranspiration of trees in relation with their architecture, their physiology and the climate. These three characteristics are determined with field measurements and data processing. Based on point clouds acquired with terrestrial laser scanner (TLS), the 3D reconstruction of the tree wood architecture is performed. Then the 3D reconstruction of leaves is carried out from the 3D skeleton of vegetative shoots and allometric statistics. With the aim of extending the simulation on several trees simultaneously, it is necessary to apply the 3D reconstruction process on each tree individually. However, as well for the acquisition as for the processing, the $3 \mathrm{D}$ reconstruction approach is time consuming. Mobile laser scanners could provide point clouds in a faster way than static TLS, but this implies a lower point density. Also the processing time could be shortened, but under the assumption that a coarser 3D model is sufficient for the simulation. In this context, the criterion of level of details and accuracy of the tree $3 \mathrm{D}$ reconstructed model must be studied. In this paper first tests to assess their impact on the determination of the evapotranspiration are presented.
\end{abstract}

\section{INTRODUCTION}

In the field of photogrammetry or more generally geomatic, modeling means representing real elements, like buildings or vegetation regarding mainly their geometry. In the field of climatology, modeling means simulating phenomena, like radiative fluxes, heat fluxes or temperature of the air based on a theoretic or semi-empirical model. In both contexts, the representation can be realized at different scales depending on the processes and the variables to be simulated.

A 3D reconstructed model which reproduces a real element is commonly characterized following 5 levels of details (LOD). For Liang et al. (2016) a tree can be described with only its height and diameter at breast or with a $3 \mathrm{D}$ reconstruction of the trunk for two different LOD (LOD 1 and 2), which represent the tree in a coarse way. In other words, the level of branches increases with the LOD, i.e. a 3D tree model with a LOD 5 contains all branches and leaves of the real tree. Concerning microclimate models, the LOD is correlated with the domain of simulation. A meso-scale model covers large regions, like for instance the Meso-NH model (Lafore et al., 1998), whereas micro-scale models focus on districts, like Envi-Met (Bruse et al., 1998). Thus meso-scale models require a coarse 3D mesh where the vegetation is taken into account in term of percentage of vegetation cover, whereas micro-scale (microclimate) models require a finer 3D mesh because trees are individualized (Vidrih and Medved, 2013) or explicitly represented in 3D through vegetative cubic boxes called "voxels" (Sinoquet et al., 2001).
Among micro-scale models, ecophysiological models that represent the microclimate inside tree crowns and tree functional responses (transpiration and photosynthesis) require a high LOD. Therefore numerous voxels with small size are used for simulating $3 \mathrm{D}$ tree geometry.

In summary, the LOD of the $3 \mathrm{D}$ tree reconstruction must be adapted to the scale of representation. Moreover, the LOD impacts the accuracy of the model, since, generally, a large scale representation will be constructed with more approximations than a small scale representation. The higher the LOD is, the higher the accuracy of the model should be. However, a low LOD (large scale) allows also time saving in data acquisition and processing.

Microclimate models need as input detailed 3D tree models. However the surveying workflow, performed in our work by terrestrial laser scanning (TLS) and the following 3D reconstruction process (from registration to skeletonization) are time consuming (Landes et al., 2015). Therefore, it is inconceivable to perform a simulation in the microclimate model with several trees at the same time without reconsidering the acquisition and processing stages leading to the 3D tree reconstructed model. Reducing acquisition time implies reducing point cloud density and accepting masks. Thus the LOD of the $3 \mathrm{D}$ reconstructed tree is impacted. On the other hand, under the assumption that the algorithm implementation has been optimized, the processing time can only be shortened if less tree details are required. In light of these observations

\footnotetext{
* Corresponding author
} 
and with the aim of extending the simulation on several trees simultaneously, we wonder whether the LOD of the 3D model of tree and its accuracy could be reduced with only low impact on the microclimate estimation.

In the next section, the approaches used in this work for microclimate and for 3D tree modeling are presented. Then, trees under study and field measurements are mentioned. Afterwards, section 4 illustrates the 3D tree reconstruction results. After that, microclimate simulation and first tests to assess the sensitivity of the estimated evapotranspiration according to the LOD of the 3D tree model are exposed. Finally, based on these first results, possible further works will be intended.

\section{MODELS USED}

\subsection{Microclimate models}

In this paper we focus on the simulation of intra tree crown microclimate modeling. Among the published ecophysiological models (Green, 1993; Sinoquet et al., 2001), the RATP model (Radiation Absorption, Transpiration and Photosynthesis) from Sinoquet et al. (2001) was chosen because it has been validated on several tree species and on both photosynthesis, transpiration rate and leaf temperature. The RATP code written in Fortran90 and Python is also available through the OpenAlea platform (Pradal et al., 2007). It was designed to simulate the spatial distribution of radiation and leaf-gas exchanges within vegetation canopies as a function of canopy structure, microclimate within the canopy and physical and physiological leaf properties. It is based on a turbid medium analogy for radiation transfers in the canopy described as a set of voxels (Figure 1). The Jarvis model and the Farquhar model are used for the leaf evapotranspiration and the leaf photosynthesis respectively. The spatial distribution of leaf temperature is estimated by closing the energy balance equation between incoming and outgoing fluxes. Concept and main equations of the RATP model are given in Sinoquet et al. (2001).

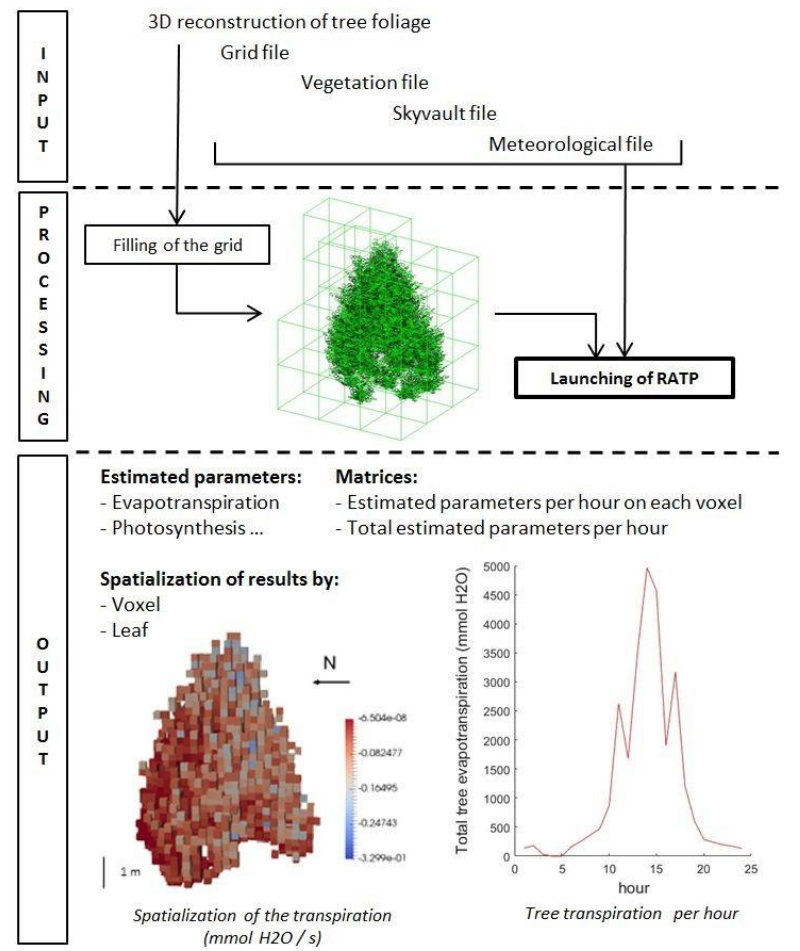

Figure 1. Concept of RATP model (input, processing, output)
As presented in Figure 1, the RATP microclimate model uses five input data.

- A 3D reconstruction of the tree foliage, characterized by the size, position and angles of leaves.

- The grid file, i.e. a matrix of voxels, integrating in each voxel the leaf area density and the tilt angles of the foliage. The size of the matrix of voxels is determined automatically according to the volume of the tree crown. The vegetation file, containing physiological parameters of tree leaves related to the stomatal conductance and the photosynthesis response functions, but also structural parameters like the clumping factor or the tilt angles of leaves.

The skyvault file, given in the literature (Den Dulk et al., 1989), integrating the discretization of the skyvault and the fraction of incident diffuse radiation.

The meteorological file, including per hour, the weather conditions around the studied tree, i.e. the global radiation, the temperature and the hygrometry of air and the wind speed.

The RATP model provides the spatial distribution within a canopy of several physiological leaf variables at a 30 to 60 minutes time step (Figure 1, bottom right). For instance, the RATP model is able to estimate the spatial distribution of the leaf evapotranspiration rate (Figure 1, bottom left). The sum of all evapotranspiration rates allows computing the whole tree evapotranspiration.

Based on allometric statistics described later (Subsection 4.3), the 3D reconstruction of the tree foliage is possible under the assumption that a detailed 3D skeleton of the leafy shoots is provided. Currently, a specific digitalization method made in the field, on the tree, is used to characterize the tree architecture. However, this laborious method which was designed for research purpose cannot be extended to practical issues i.e. when the architecture of large trees or several trees are needed. That is why a new and faster method for acquiring this tree architecture has been developed and is presented in next section.

\subsection{D models of tree architecture}

2.2.1 Related works: In the literature, two automatic methods for point clouds generation can be found. The first one uses photogrammetric techniques based on the processing of terrestrial photographs and the second one uses laser scanning techniques, like the use of TLS. Although both techniques are able to provide point clouds, much more points of view are required by photogrammetry, because of the tree architecture complexity. Therefore, the laser scanner seems to be the most adequate sensor for acquiring in fast way point clouds over several trees.

Concerning models for 3D tree reconstruction, several studies based on point cloud processing exist. On the one hand there are models based on the adjustment of tree point cloud by geometric primitives, e.g. "SimpleTree" developed by Hackenberg et al. (2015). On the other hand, there are models that introduce the skeleton concept for describing geometry of trees in a relevant way, as the "SkelTree" model developed by Bucksch et al. (2010). In our study, the skeletonization is chosen because it facilitates the determination of the required parameters for tree foliage reconstruction, i.e. length and spatialization of the branches and shoots. Among the published skeletonization methods, the Cao et al. (2010) algorithm was 
chosen since it seems to be less sensitive than the others to noisy data, as TLS point clouds are known to be, especially on thin parts like tree branches.

2.2.2 Developed model: The 3D tree reconstruction model developed in this study is inspired from Cao et al. (2010) and has been developed in Matlab. The algorithm aims to reconstruct an element with a $3 \mathrm{D}$ curve skeleton. The original version of Cao et al. (2010) is composed of two steps: a geometric contraction of the point cloud with a local Delaunay triangulation, followed by a topological thinning. A drawback of this method is that for reconstructing a whole tree with complex architecture, it is necessary to perform a previous segmentation in order to isolate each complex part. In our case, it means that every extremity of a branch which bears annual vegetative shoots must be isolated.

Given that the algorithm proposed by Cao et al. (2010) does not fulfill these requirements, several improvements have been carried out. The final processing steps developed in our work are mentioned in Figure 2. Some false skeleton nodes have been removed in the steps denoted as "trunk reconstruction" and "removal of nodes". Finally skeleton nodes have been classified according to the shoots they belong to and ordered from the stump center to the shoot extremity. The classification step is necessary for the foliage reconstruction step (Subsection 4.3). More information about the reconstruction processing chain can be found in Landes et al. (2015).

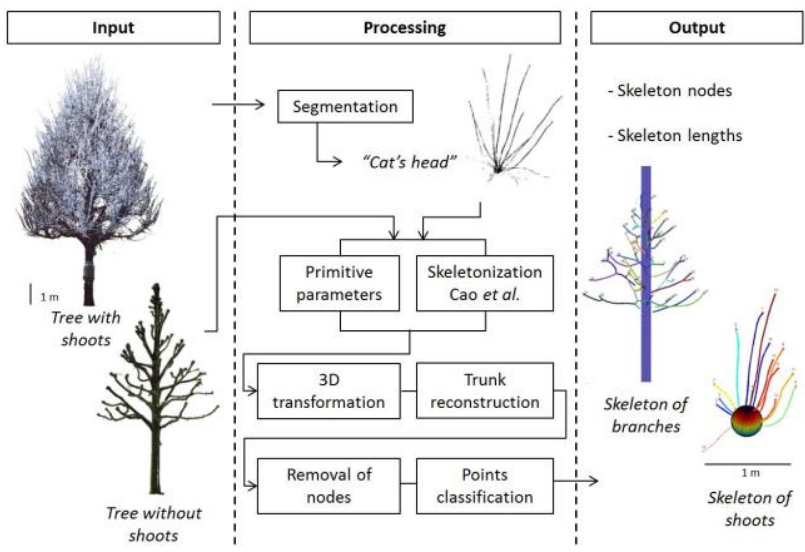

Figure 2. Concept of the 3D tree reconstruction processing chain

The input of our processing chain are, as illustrated in Figure 2, the point cloud of the tree with shoots and the point cloud of the tree without shoots. As the aim of the process is to produce a 3D reconstruction of all branches and all shoots of the tree under study, each segmented complex part, called "cat's head" (Figure 3) is also an input of the skeletonization algorithm.

Despite a few residual errors in the 3D skeletons of ligneous tree elements, and some manual interventions during the processing, the $3 \mathrm{D}$ tree model produced by the developed reconstruction algorithm is a much faster solution for acquiring the tree architecture than the current digitalization method carried out directly on the tree in the field. This is particularly important in the purpose of extending the 3D tree reconstruction to several trees simultaneously. This skeletonization modeling will be used to assess the impact of LOD of the 3D tree model on the evapotranspiration, estimated by RATP microclimate model. First of all, the trees under study and the sensors used for our work must be described.

\section{TREES UNDER STUDY}

Since 2013, a field experiment is conducted in the city of Strasbourg (France) to explore the role of urban vegetation on urban climate. A network of sensors distributed in 25 different sites has been established (Najjar et al., 2015). For our tests we focused on ten silver lime trees located in the garden of the "Palais universitaire". As illustrated in Figure 3, the foliage of a silver lime tree appears in April and remains until October. These trees have the particularity to be pruned in "cat's head" shape by the gardeners in January/February. As a consequence, at the extremity of the pruned branches, a complex ligneous mass resulting from repeated pruning, called "stump" is formed. Most shoots grow on these stumps. In this paper we call "cat's head" the whole extremity of a branch, i.e. the stump as well as its shoots. Thus, silver lime trees can be observed at several phenological stages: as a pruned tree, as a leafy tree and with shoots only (Figure 3). Several point cloud acquisitions of these three phenological stages have been performed during the years, since the tree geometry is completely different at every stage. Consequently, three different 3D tree models have been produced.
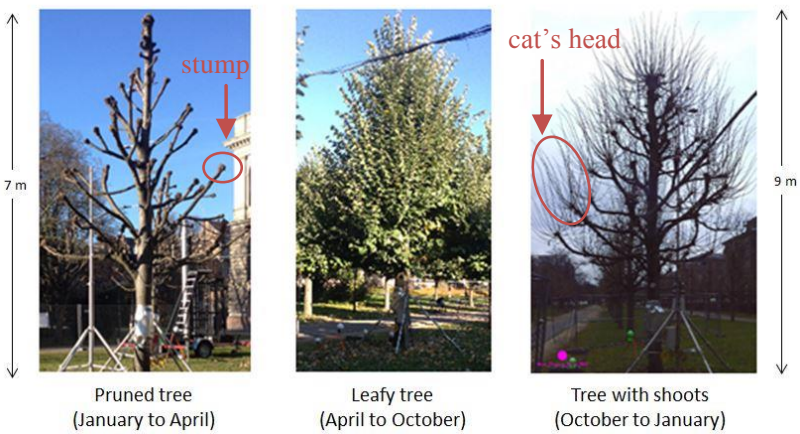

Figure 3. Illustration of the three phenological stages of a silver lime tree

\subsection{Field measurements for providing RATP inputs}

On six of these silver lime trees and in their surroundings, several measurements are continuously registered (Najjar et al., 2015) and some of them are detailed in Table 4. Based on these field measurements, three input files of RATP model can already be determined: the 3D reconstruction of tree foliage, the vegetation file and the meteorological file.

The climatological data are continually recorded with 3 sensors on a 20 meters mast and 3 other sensors located on the roof $(300 \mathrm{~m})$ of a neighboring building. Concerning the leaf physiological data, a LI-COR 6400 was used to measure gas exchanges between plant and environmental variables which control stomatal conductance of leaves. For the allometric statistics related to the position, size and orientation of leaves on shoots, manual measurements were carried out on leafy shoot samples. Finally, a TLS campaign allows the acquisition of the tree point clouds. A description of the surveying workflow is presented in Subsection 3.2.

Other field measurements are made for the validation step of the outputs (estimations) of our microclimate model. Concerning the RATP estimation of the total tree evapotranspiration, it can be assumed as equivalent to the total flux of water within sapwood obtained with sap flow sensors. This measurement is also illustrated in the last row of Table 4 and described in Ngao et al. (2015). 


\begin{tabular}{|c|c|c|c|}
\hline Sensor & Picture & Extracted data & Data usage \\
\hline $\begin{array}{c}3 \text { sensors on a } \\
\text { mast }(20 \mathrm{~m})\end{array}$ & & $\begin{array}{c}\text { Air temperature } \\
\text { Floor temperature } \\
\text { Air humidity } \\
\text { Wind speed } \\
\mathrm{CO} 2_{\text {air }}\end{array}$ & $\begin{array}{l}\text { Meteorological } \\
\text { input data }\end{array}$ \\
\hline $\begin{array}{l}3 \text { sensors on a } \\
\text { roof }(300 \mathrm{~m})\end{array}$ & & $\begin{array}{c}\text { PAR, NIR } \\
\text { (global/diffuse) } \\
\text { and atmospheric } \\
\text { radiation }\end{array}$ & $\begin{array}{l}\text { Meteorological } \\
\text { input data }\end{array}$ \\
\hline LI-COR 6400 & & $\begin{array}{c}\text { Gas exchanges }=> \\
\text { Stomatal } \\
\text { conductance }\end{array}$ & $\begin{array}{c}\text { Leaf gas } \\
\text { exchange } \\
\text { parameters }\end{array}$ \\
\hline $\begin{array}{c}\text { Manual } \\
\text { measurements } \\
\text { on leafy shoots } \\
\text { sample }\end{array}$ & & $\begin{array}{c}\text { Allometric } \\
\text { statistics } \\
\text { (number/area/ } \\
\text { angles of leaves) }\end{array}$ & $\begin{array}{c}\text { Leaf } \\
\text { reconstruction }\end{array}$ \\
\hline $\begin{array}{l}\text { Terrestrial Laser } \\
\text { Scanner (TLS) }\end{array}$ & & Point clouds & $\begin{array}{l}3 \mathrm{D} \text { tree } \\
\text { reconstruction }\end{array}$ \\
\hline $\begin{array}{c}2 \text { T-type } \\
\text { thermocouples }\end{array}$ & & Sap flow & $\begin{array}{l}\text { Evapotranspi- } \\
\text { ration } \\
\text { comparison }\end{array}$ \\
\hline
\end{tabular}

Table 4. Field measurements useful for RATP with corresponding sensors

\subsection{Surveying workflow for providing tree architecture geometry}

To acquire the tree architecture geometry of the silver lime trees located in the garden, a surveying workflow has been carried out and repeated for each phenological stage of the tree. Several point clouds have been acquired around the trees with a TLS from FARO (Focus 3D X 330). With the settings of the TLS parameters presented in Table 5, 15 stations have been established around the 10 trees, along 3 rows, in order to consider more than 4 viewpoints for every tree.

\begin{tabular}{|l|c|c|c|}
\hline \multirow{2}{*}{ Parameters } & \multicolumn{3}{|c|}{ Tree phenology } \\
\cline { 2 - 4 } & $\begin{array}{c}\text { Pruned } \\
\text { tree }\end{array}$ & Leafy tree & $\begin{array}{c}\text { Tree with } \\
\text { shoots }\end{array}$ \\
\hline $\begin{array}{l}\text { Spacing between points } \\
\text { (at 10 } \text { m from one station) }\end{array}$ & $12 \mathrm{~mm}$ & $30 \mathrm{~mm}$ & $6 \mathrm{~mm}$ \\
\hline Field of view & \multicolumn{3}{|c|}{$300^{\circ} / 360^{\circ}$} \\
\hline R,G,B (TLS camera) & \multicolumn{3}{|c|}{ Yes } \\
\hline Acquisition time / station & $5 \mathrm{~min}$ & $3 \mathrm{~min}$ & $10 \mathrm{~min}$ \\
\hline
\end{tabular}

Table 5. Settings defined during laser scanning campaigns, for each phenological stage.
Several pre-processing steps must be considered before obtaining a point cloud usable for $3 \mathrm{D}$ reconstruction. Based on spherical targets, adequately positioned in the field before the scanning, the registration of the point cloud stations into one unique point cloud is performed (with $4 \mathrm{~mm}$ accuracy). Based on a reference network of control points, tacheometric measurements allowed the georeferencing of the whole point cloud. The georeferencing is crucial for ensuring proper estimations of the RATP model, since it needs the exact tree geolocalisation (latitude and longitude) for computing the exact sun trajectory. Then, after a segmentation of each tree point cloud, a de-noising step is carried out.

With the aim to reconstruct an accurate 3D model of a tree and studying the effect of the LOD, 4 scanning stations with high density must be carried out around every tree. In this paper, the $3 \mathrm{D}$ reconstruction process and the estimations of the microclimate model focus only on one of the ten trees. Our algorithms must first be validated on one tree before extending their application to several silver lime trees.

\section{RESULTS OF 3D TREE RECONSTRUCTIONS}

In order to assess the impact of LOD in the 3D reconstruction of trees for microclimate modeling, four 3D models of the ligneous parts (shoots) of one silver lime tree have been produced. Figure 6 presents the four different models with a focus on the same cat's head. The particularity of these models is that the same tree is reconstructed following different LOD, with a decreasing accuracy from LOD 6 to LOD 1.

In our paper, we relate the LOD of the tree model to the LOD of the shoots, which characteristics are presented in Table 7 . We consider that a LOD 3 with estimated position, number and length of shoots has a higher accuracy than a LOD 2 containing false parameters.

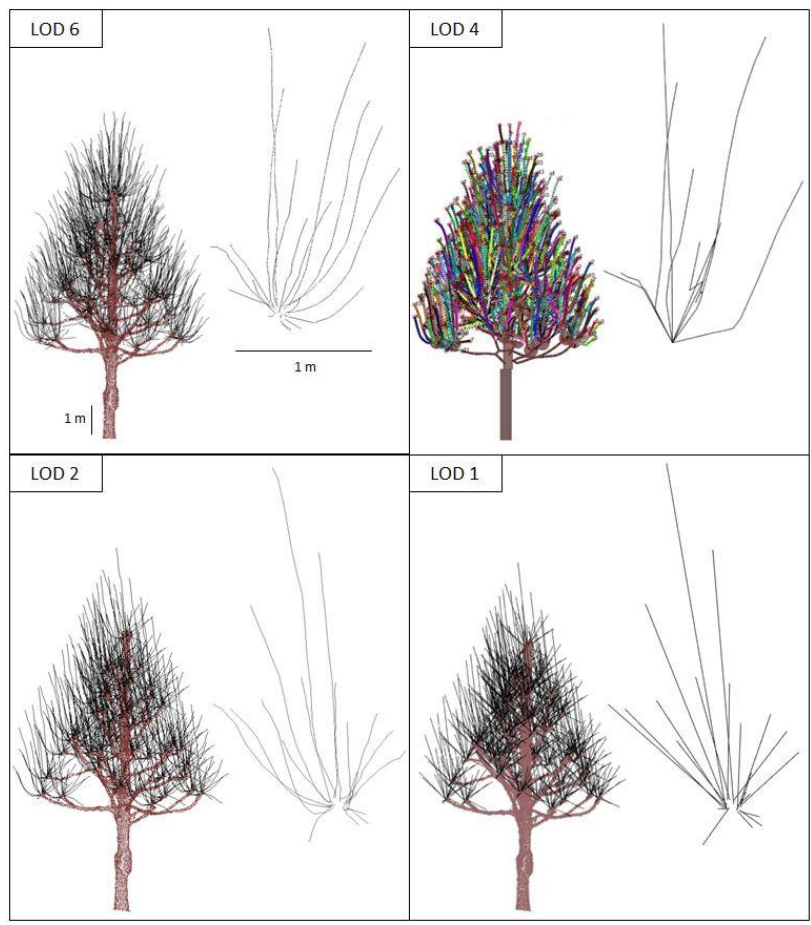

Figure 6. 3D tree models with different LOD (tree on the left and cat's head on the right) 


\begin{tabular}{|c|c|c|c|c|}
\cline { 2 - 5 } \multicolumn{1}{c|}{} & Position & Number & Length & Curvature \\
\hline LOD 6 & True & True & True & Curve \\
\hline LOD 5 & True & True & False & Curve \\
\hline LOD 4 & True & False & False & Curve \\
\hline LOD 3 & Estimated & Estimated & Estimated & Curve \\
\hline LOD 2 & False & False & False & Curve \\
\hline LOD 1 & False & False & False & Straight \\
\hline
\end{tabular}

Table 7. Characteristics of each LOD of a 3D tree model of shoots

Four of these six LOD models have been created and the way followed to construct them is explained in next subsection.

\subsection{D shoot reconstructions}

The spatialization of the shoots, since they are support of the tree leaves, is needed for foliage reconstruction. That is why the $3 \mathrm{D}$ reconstruction process must be applied on every cat's head.

The LOD 6 model is assumed to be a "reference model" of the tree shoots, because it has been produced based on a manual digitalization in the point cloud. First, a segmentation of each 52 cat's head of the tree has been performed. Then, based on every cat's head point cloud, a digitalization of each shoot, by picking point from one extremity to the stump has been carried out. This processing offers an accurate 3D curve skeleton of all the tree shoots which are located on each stump. Length of each shoot of 5 cat's heads samples have been measured in the field. Results show that each shoot length of the reference model presents in average a deviation of $6 \mathrm{~cm}+/-11 \mathrm{~cm}$ from the exact value. Obviously, even if this model is very useful for research purpose and for the assessment of our automatic reconstruction process, it requires 50 hours of processing. This is not acceptable for fast acquisition of several tree architectures.

The LOD 4 model is created with our developed 3D tree reconstruction algorithm (see Subsection 2.2). After a segmentation of the cat's heads and the stumps, the center of every stump is calculated by arithmetic average of the points covering it. Then, the automatic skeletonization algorithm is used. Since this algorithm provides a 3D curve skeleton with some false skeletons, this second model can be considered as less accurate than the LOD 6. As described in the introduction, reducing the accuracy of the $3 \mathrm{D}$ tree reconstruction allows the shortening of the processing time, by half with about 23 hours, but it is still not acceptable in the purpose of several trees reconstruction.

The third model is produced with a lower LOD (LOD 2) and therefore a lower accuracy. The processing time is also reduced. In this case, one representative cat's head has been manually segmented and digitalized. This 3D curve skeleton is then duplicated on the 52 centers of stumps extracted previously. This operation requires 2 hours and 30 minutes processing time, which is acceptable if we want to apply this reconstruction process on several trees. However, the accuracy of the model is very impacted. Shoots position and orientation make the tree crown unrealistic.

Finally, the LOD 1 model is created based on the LOD 2 model. This model considers straight shoots instead of curve shoots. For this purpose, only two points are taken into account for one shoot, i.e. the extremity and the connection to the stump. With the same processing time as the LOD 2 model, it allows to evaluate the impact of a model with straight shoots.

\begin{tabular}{|c|c|c|c|c|c|}
\hline & \multicolumn{2}{|c|}{ Shoot } & \multirow{2}{*}{ Processing } & \multirow{2}{*}{\multicolumn{2}{|c|}{$\begin{array}{c}\begin{array}{c}\text { Time } \\
\text { (hours) }\end{array} \\
\end{array}$}} \\
\hline & Number & Length (m) & & & \\
\hline \multirow{2}{*}{$L O D 6$} & \multirow{2}{*}{1077} & \multirow{2}{*}{982.6} & Segmentation & 21 & \multirow{2}{*}{50} \\
\hline & & & Digitalization & 29 & \\
\hline \multirow{3}{*}{$L O D 4$} & \multirow{3}{*}{759} & \multirow{3}{*}{888.7} & Segmentation & 21 & \multirow{3}{*}{23} \\
\hline & & & Stump segmentation & 1 & \\
\hline & & & Skeletonization & 1 & \\
\hline \multirow{4}{*}{$L O D 2$} & \multirow{4}{*}{1092} & \multirow{4}{*}{851.0} & Segmentation & $1 / 2$ & \\
\hline & & & Digitalization & $1 / 2$ & \\
\hline & & & Stump segmentation & 1 & \\
\hline & & & Duplication & $1 / 2$ & \\
\hline$L O D 1$ & 1092 & 822.2 & Identical to LOD & & 2.5 \\
\hline
\end{tabular}

Table 8. Characteristics of the four LOD models

Table 8 presents the results of the four models regarding the total length and number of reconstructed shoots. Moreover, the processing steps and the processing time required for their reconstruction are detailed. It shows that, in addition to different processing times, the different models provide neither the same number nor the same total length of shoots. The total length reaches in average for the LOD 4, 2 and 1 models about $13 \%$ of the reference model. Concerning the total number of shoots, only the LOD 4 model has a significant difference with the reference model, with $30 \%$ of missing shoots, because of the false skeletons. The impact of the LOD on the 3D foliage reconstruction will be presented in Subsection 4.3.

\subsection{D shoot reconstructions according to the season}

The leafy crown of our silver lime trees is not as big in June as in October, because the volume of the tree crown increases during this period. Therefore the number of leaves also increases. This impacts the evapotranspiration rate of the tree. Thus, if an estimation of the evapotranspiration in June or in October is desired, it is necessary to construct respectively the $3 \mathrm{D}$ tree model when the volume of the leafy tree crown is the lowest and the highest.

In this paper, RATP simulations have been carried out on a four months period, from June $13^{\text {th }}$ to September $29^{\text {th }}, 2014$. For the tests we want to perform with the microclimate model, two foliages are necessary, the "June" foliage for the first half of the studied period and the "October" foliage for the second half period. For the moment, the four reconstructed 3D tree models concern October. Concerning June, given that shoots are visible only after the leaf fall, the skeletonization based on point clouds acquired in June is not possible. That is why another method was used for reconstructing the shoots of June.

Based on the four skeletons representing the tree in October and the leafy tree point cloud in June, four new models related to the tree in June can be computed with a Matlab script. From the tree point cloud of June, a convex hull of the tree crown is created. Then, as illustrated in Figure 9a, all the nodes of the 3D curve skeletons of the October model (in brown), which are outside the convex hull (in green), are removed from the skeleton. As a consequence, 3D curve skeletons used for the creation of the June foliage are about $20 \%$ smaller than for the October foliage. Thus, even if this reconstruction method was created under the assumption that it respects the real structure of June shoots, it allows keeping the knowledge of their spatialization. Thus, for the following RATP tests, each model characterised by a LOD has been produced with two tree shoot reconstructions (June and October). 


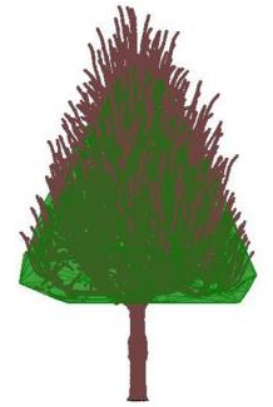

a)

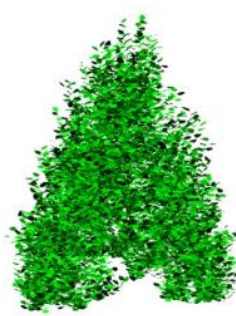

b)

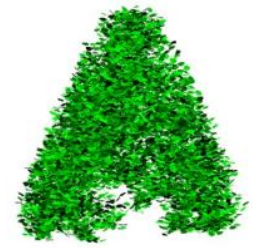

c)
Figure 9. Substraction of models for June foliage creation (a); results of $3 \mathrm{D}$ reconstructed foliage

of October (b) and June (c)

\subsection{D foliage reconstructions}

The 3D reconstruction of the foliage is directly an input for the RATP model, as described in Subsection 2.1. Based on field measurements presented in Subsection 3.1, allometric statistics related to the spatialization of leaves on the shoots are determined with the Sonohat et al. (2006) method. From the 3D skeleton of vegetative shoots and the knowledge of these allometric statistics, leaves are reconstructed in 3D (Figure 9b and $9 \mathrm{c})$. The foliage reconstruction is very detailed with the estimation of number, position, orientation and shape of the leaves on each shoot.

Shoot length is a very important parameter for the foliage reconstruction, since the number of leaves estimated in a shoot depends on its length. As already mentioned, the different 3D models obtained for the tree shoots do not have the same number and total length of shoots. The impact of these characteristics on the 3D foliage reconstructions (Figure 9b and $9 \mathrm{c})$ is visible with the computed total leaf area by tree. Indeed, the leaf area of the four October models are in average reduced by $13 \%$ when comparing LOD 4,2 and 1 to LOD $6\left(234 \mathrm{~m}^{2}\right)$. Moreover, the difference of leaf surface between June and October is about $+12 \%$.

At this stage, we can start the analysis of the impact of different total leaf surfaces and of leaves positioning on the estimated evapotranspiration.

\section{ANALYSIS OF THE EVAPOTRANSPIRATION}

\subsection{RATP estimation compared with other measurements}

Based on each foliage reconstruction (see Figure $9 \mathrm{~b}$ and $9 \mathrm{c}$ ) and on the four other input parameters of RATP model (see Subsection 2.1), the algorithm can operate and supply realistic estimations of the evapotranspiration.

To assess the accuracy of this estimated parameter, the method described in Ngao et al. (2015), with the sap flow sensor, is used. Given that the accuracy of the sap flow measurements is under determination, only a comparison between the evapotranspiration estimated with this sensor and computed with RATP is carried out here. For this assessment, the reference model (LOD 6) has been chosen for its high accuracy. Moreover, a voxel resolution of 50 centimeters is chosen for the grid file (input of RATP), in adequacy with the leaf area of a silver lime tree and in order to have neither too little nor too much leaves in a voxel (see "processing stage" in Figure 1). Figure 10 represents the total evapotranspiration by day estimated with RATP following the estimation with the flux of sap flow. The correlation curve (red) is obtained with the evapotranspiration rates estimated with the LOD 6 model (red crosses). It allows seeing a trend with an underestimation of RATP when the evapotranspiration is lower than about $4.10^{4}$ mmol H2O/day, and an overestimation of RATP for higher values. There is a difference of $21 \%$ between the two estimations with a standard deviation of $+/-36 \%$.

These results are not as good as works already published using the RATP model (Sinoquet et al., 2001). However they can be explained by the parametrization of the RATP input data. Indeed, first of all some stomatal functions according to the silver lime tree were not fully determined. Then the radiative radiation used in the meteorological file does not take into account building and vegetation shadows. Thus, in order to improve the accuracy of the estimations made by the microclimate model, it is necessary to focus on the improvement of the input data. As in this paper the objective is the evaluation of the impact of LOD of the 3D tree reconstruction on microclimate modeling, the current parametrization of RATP model is largely sufficient.

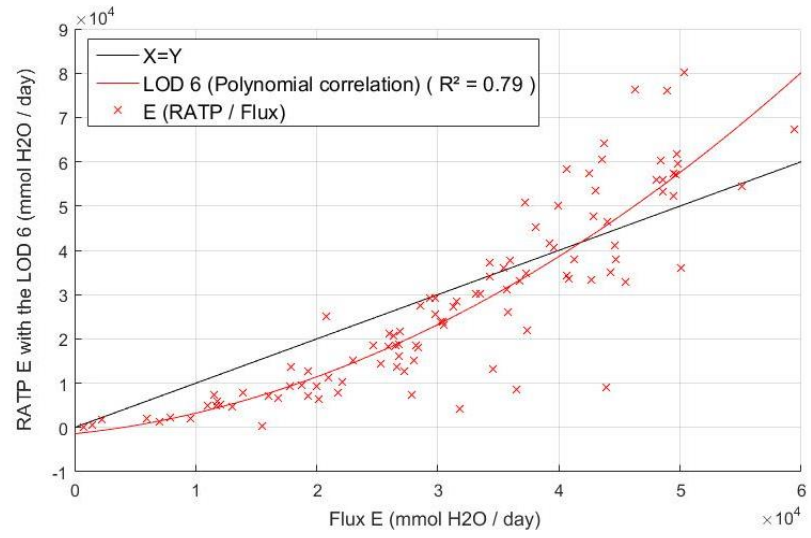

Figure 10. Comparison of the evapotranspiration (E) estimated by RATP model, with the flux of sap flow

\subsection{Impact of LOD}

The assessment of the sensitivity of the evapotranspiration estimated by RATP according to the LOD of the 3D tree model is carried out in this subsection with a voxel size of $50 \mathrm{~cm}$. As already mentioned in Subsection 4.2, the four models (LOD 6, LOD 4, LOD 2 and LOD 1) used for this purpose are composed of two different foliages (in June and in October).

Each foliage is characterized by different positions and tilt angles of leaves. This influences the clumping factor. It must be noticed that the clumping factor is very important in the evapotranspiration estimation provided by the RATP model. It quantifies the aggregation of leaves within each voxel and enables the right estimation of the radiation intercepted by group of leaves in each voxel (Sinoquet et al., 2007). The difference regarding the clumping factor between the four models is low. Indeed, it lies between 0.50 and 0.58 (on a scale of 0.2 to nearly 1.2 ) with always a lower value for the June foliage than the October foliage. This observation allows assuming that leaves in June are more clustered than in October. 
Figure 12 illustrates the evapotranspiration estimated by day according to the four LOD models used: in red the LOD 6, in blue the LOD 4, in green the LOD 2 and in black the LOD 1. The results of the evapotranspiration estimated per day according to the LOD models show that in average there are significant differences, in absolute values, between the LOD 6 and the LOD 4 (about $6 \%+/-7 \%$ ), the LOD 2 (about $13 \%+/-$ $12 \%)$ and the LOD $1(15 \%+/-13 \%)$. Moreover, by summing the evapotranspiration rate of all the days, the tree evapotranspiration during the vegetative shoot period, i.e. 4 months from June to October is obtained. The sensitivity of the evapotranspiration between LOD 4-2-1 and LOD 6 is presented in Table 11. We can affirm that usually the coarser the LOD of the $3 \mathrm{D}$ tree model is, the lower the evapotranspiration is estimated by RATP.

\begin{tabular}{|l|c|c|c|}
\cline { 2 - 4 } \multicolumn{1}{c|}{} & $\begin{array}{c}\text { Two } \\
\text { foliages }\end{array}$ & $\begin{array}{c}\text { June } \\
\text { foliage }\end{array}$ & $\begin{array}{c}\text { October } \\
\text { foliage }\end{array}$ \\
\hline$L O D 4 / L O D 6$ & $-2 \%$ & $2 \%$ & $-6 \%$ \\
\hline$L O D$ 2 / LOD 6 & $-9 \%$ & $0 \%$ & $-20 \%$ \\
\hline$L O D$ 1 / LOD 6 & $-12 \%$ & $-3 \%$ & $-22 \%$ \\
\hline
\end{tabular}

Table 11. Sensitivity of the evapotranspiration on the whole period depending on the LOD and the foliage used

The curves presented in Figure 12 reveal also that the sensitivity between the different LOD is not equal before and after the $8^{\text {th }}$ of August, 2014. This date corresponds to the application of the June foliage in the first half of the period and of the October foliage in the second half of the period. Two other tests were carried out in order to better understand the origin of this difference in the evapotranspiration estimate. RATP estimation was tackled on the whole period (4 months), but only with the "June foliage" for the first test and the "October foliage" for the second test. Assumed that the processing used to create the June foliage reconstructs the reality, the results presented in Table 11 allow to conclude that the difference of sensitivity between the beginning and the end of the period is due to structural parameters of foliages. Indeed, with the June foliage, the leaves are so much clustered (traduced by a small clumping factor), that the differences of evapotranspiration estimated from the different LOD tree models are almost null. Therefore differences between the two structures of foliage seem to have more impact on the estimation of the evapotranspiration than the LOD have.

The sensitivity of the evapotranspiration estimate to the LOD seems also different according to the day. As we can see on Figure 12 (zoom), the sensitivity observed for September $1^{\text {st }}$ is lower than for September $2^{\text {nd }}$. This phenomenon is due to the weather conditions for these days. Indeed, during sunny day characterized by a high global radiation $(02 / 09)$, the tree evapotranspiration is bigger. Therefore, the geometry of the foliage has more impact on the result during a sunny day than during a cloudy day (01/09), when the radiation is twice lower. But, despite the heterogeneous weather conditions during the four months studied, it is interesting to notice that for the totality of the evapotranspiration estimated during the period, the sensitivity to LOD is low with a maximum of $-12 \%$ (Table $11)$.

\subsection{Results interpretation}

The previous experimentations allow to conclude that evapotranspiration estimated by RATP is not very sensitive to the reduction of the LOD of the $3 \mathrm{D}$ tree model. Concerning the characteristics of the LOD tree models (Table 7), the real position and orientation of shoots seem to have more impact on the evapotranspiration computation than the curvature of shoots. Moreover, the foliage structure plays also an important role in the sensitivity of the estimation. Therefore, it will be necessary to determine the effect of some parameters related to the tree like the leaf area density (LAD), the clumping factor or the leaf area index (LAI) on the evapotranspiration rate. Finally, there is a variability of the evapotranspiration estimation according to the weather factor (especially the radiation) driving the evapotranspiration process.

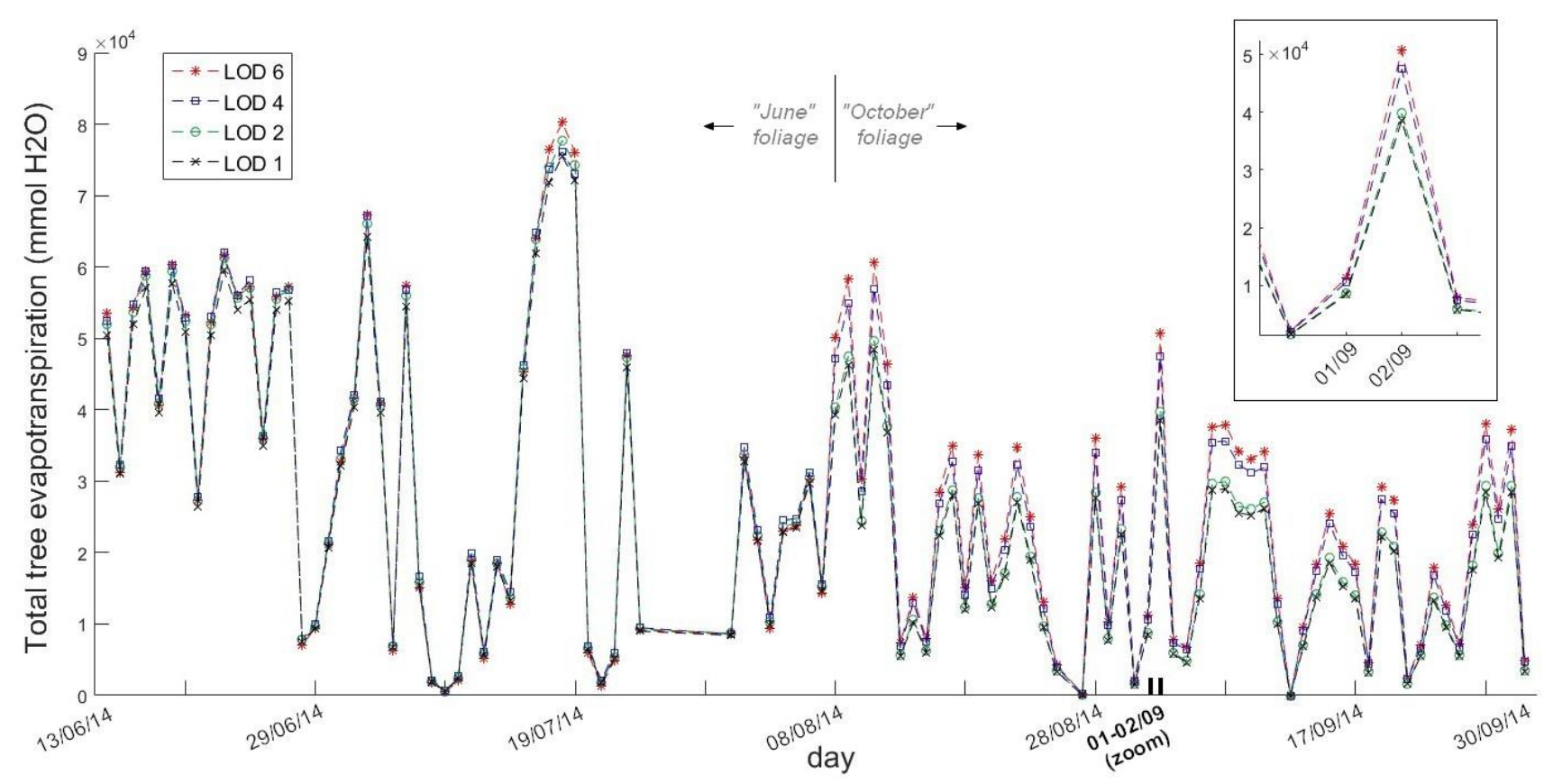

Figure 12. Sensitivity of the evapotranspiration by day depending on the LOD of the tree 3D model 


\section{CONCLUSION}

In this paper, we have evaluated the impact of the LOD of a 3D tree model on the tree evapotranspiration estimated by a microclimate model RATP. This sensitivity study will help us to find a way to extend the simulation on several trees simultaneously, in order to assess their cooling effect, while ensuring a reasonable processing time.

Based on TLS point clouds, four LOD of 3D tree models have been reconstructed. They allowed the determination of two different structures of foliage by model, in order to calculate evapotranspiration estimations during 4 summer months. The first tests aiming to assess the impact of the reduction of the LOD (correlated with the accuracy of the 3D tree model) on the evapotranspiration provide interesting results. Indeed, the total evapotranspiration estimated with the lowest LOD (LOD 1) and the lowest accuracy (straight shoots with false position, length and number) presents a difference of $12 \%$ with the estimation obtained with the highest LOD (LOD 6). In parallel the processing time for the LOD 6 reconstruction is reduced by a factor 20 when the LOD 1 is used.

These results show that the detailed knowledge of the structure of the shoots bearing leaves is probably not necessary for modeling urban tree cooling. Therefore, estimations with a less accurate model of the tree structure, i.e. a LOD 0 , derived from the convex hull of the leafy tree with some additional information about the foliage structure (LAD, LAI, clumping) should be carried out.

This work is a first step and will be continued based on mobile LiDAR data that could be used for this coarse 3D reconstruction. Despite of limitations deriving from hypothesis and non-estimated parameters in the evapotranspiration modeling, this work emphasizes the complexity in dealing with trees and atmosphere interactions where both structural, climatic and functional processes are involved. To provide guidelines to urban planners for introducing trees in cities, it is necessary to unravel the role of each component and this work contributes to this objective.

\section{ACKNOWLEDGEMENTS}

The authors would like to thank Jérôme Ngao, INRA member for providing sap flow data, and Marine Lempereur, INSA master student, for realizing the third 3D tree model (LOD 2).

\section{REFERENCES}

Liang, X., Kankare, V., Hyyppä, J., Wanga, Y., Kukko, A., Haggrén, H., Yu, X., Kaartinen, H., Jaakkola, A., Guan, F., Holopainen, M., and Vastaranta, M., 1998. Terrestrial laser scanning in forest inventories. ISPRS Journal of Photogrammetry and Remote Sensing, 115, pp. 63-77.

Lafore, J. P., Stein, J., Asencio, N., Bougeault, P., Ducrocq, V., Duron, J., Fischer, C., Héreil, P., Mascart, P., Masson, V., Pinty, J. P., Redelsperger, J. L., Richard, E., and Vilà-Guerau de Arellano, J., 1998. The Meso-NH Atmospheric Simulation System. Part 1. Ann. Geophysicae, 16, pp. 90-109.

Bruse, M., and Fleer, H., 1998. Simulating surface-plant-air interactions inside urban environments with a three dimensional numerical model. Environ. Modell. Softw. 13, pp. 373-384.
Vidrih, B., and Medved, S., 2013. Multiparametric model of urban park cooling island. Urban Forestry \& Urban Greening, 12 , pp. $220-229$.

Sinoquet, H., Le Roux, X., Adam, B., Ameglio, T., and Daudet, F.A., 2001. RATP: a model for simulating the spatial distribution of radiation absorption, transpiration and photosynthesis within canopies: application to an isolated tree crown. Plant, Cell \& Environment, 24, pp. 395-406.

Landes, T., Saudreau, M., Najjar, G., Kastendeuch, P., Guillemin, S., Colin, J., and Luhahe, R., 2015. 3D tree architecture modeling from laser scanning for urban microclimate study. In Proceedings of the 9th International Conference on Urban Climate jointly with 12th Symposium on the Urban Environment, Toulouse, France.

Green, S.R.,1993. Radiation balance, transpiration and photosynthesis of an isolated tree. Agricultural and Forest Meteorology, 64, pp. 201-221.

Pradal, C., Dufour-Kowalski, S., Boudon, F., Fournier, C., Godin, C., 2008. OpenAlea: a visual programming and component-based software platform for plant modelling. Functional Plant Biology, 35(9-10), pp751-760.

Den Dulk, J.A., 1989. The interpretation of remote sensing, a feasibility study. Thesis, Wageningen University, in Netherlands.

Hackenberg, J., Spiecker, H., Calders, K., Disney, M., and Raumonen, P., 2015. SimpleTree - An efficient open source tool to build tree models from TLS clouds. Forests, 6, pp. 4245-4294.

Bucksch, A., Lindenbergh, R., and Menenti, M., 2010. SkelTre. The Visual Computer, 26, pp. 1283-1300.

Cao, J., Tagliasacchi, A., Olson, M., Zhang, H., and Su, Z., 2010. Point cloud skeletons via Laplacian-based contraction. In Proceedings of the IEEE International Conference on Shape Modeling and Applications, Aix en Provence, France, pp.187197.

Najjar, G., Colin, J., Kastendeuch, P., Ngao, J., Saudreau, M., Landes, T., Améglio, T., Luhahe, R., Guillemin, S., Schreiner, G., Kleinpeter, J., and Nerry, F., 2015. A three years long fieldwork experiment to monitor the role of vegetation on the urban climate of the city of Strasbourg, France. In Proceedings of the 9th International Conference on Urban Climate jointly with 12th Symposium on the Urban Environment, Toulouse, France.

Ngao, J., Colin, J., Améglio, T., Saudreau, M., Kastendeuch, P., Granier, A., and Najjar, G., 2015. Temporal variations of transpiration and latent heat fluxes from isolated crowns and lawns in a park at Strasbourg, France. In Proceedings of the 9th International Conference on Urban Climate jointly with 12th Symposium on the Urban Environment, Toulouse, France.

Sonohat, G., Sinoquet, H., Kulandaivelu, V., Combes, D., and Lescourret, F., 2006. Three-dimensional reconstruction of partially 3D-digitized peach tree canopies. Tree Physiology, 26, pp. 337-351.

Sinoquet, H., Stephan, J., Sonohat, G., Lauri, P.E., Monney, P., 2007. Simple equations to estimate light interception by isolated trees from canopy structure features: assessment with threedimensional digitized apple trees. New Phytologist, 175(1), pp 94-106. 